\title{
NUMERICAL STUDY OF COOLING BY TANGENTIAL SYNTHETIC JET
}

\author{
F. Munhoz, ABSTRACT \\ C. Y. Y. Lee, \\ and F. L. D. Alves \\ Universidade do Vale do Rio dos Sinos \\ Programa de Pós-Graduação em Engenharia \\ Mecânica \\ Bairro Cristo Rei \\ CEP 93022-000, São Leopoldo, RS, Brasil \\ conrady@unisinos.br \\ Modern electronics are becoming more compact and with higher processing \\ power, which translates into a demand for higher heat dissipation. Current \\ electronic "coolers," which are based on the combination of fans and heat \\ sinks, are becoming unable to provide sufficient heat dissipation since they \\ rely primarily on generating large volumetric flowrates of air to achieve \\ their results. As an alternative, synthetic jets are under consideration due to \\ their known property to enhance turbulence and heat transfer. Synthetic jets \\ are produced by the oscillation of a membrane in a sealed cavity equipped \\ with an orifice. For this study, a numerical model of channel mounted with \\ a heating element on one surface and a synthetic jet directed to blow along \\ the wall was constructed on ANSYS CFX. Heat dissipation provided by the \\ synthetic jet was analyzed with respect to changes in Reynolds number, \\ pulsing frequency and placement of the heated element. Results were \\ compared to a conventional technique represented by a steady channel flow \\ of equivalent mass flow rate to the average flow induced by the synthetic \\ jet. Results showed that the synthetic jet formed a thin layer of intense \\ vorticity along the targeted surface with cooling greatly outperforming \\ conventional techniques. Synthetic jet cooling was also determined to be \\ most affected by jet velocity and Reynolds number while pulsing frequency \\ and placement of the heated element were not as influential. \\ Received: January 12, 2015 \\ Revised: February 23, 2015 \\ Accepted: March 31, 2015 \\ Keywords: synthetic jets, Reynolds number, electronic cooling devices
}

\section{NOMENCLATURE}

amplitude of velocity oscillation at the throat entrance, $\mathrm{m} / \mathrm{s}$

$\mathrm{C}_{\mathrm{p}}$ fluid specific heat at constant pressure, $\mathrm{J} /(\mathrm{kg} \cdot \mathrm{K})$

$\mathrm{d} \quad$ length of the heated element, $\mathrm{m}$

f pulsing frequency, $\mathrm{Hz}$

h convective heat transfer coefficient, $\mathrm{W} /\left(\mathrm{m}^{2} \cdot \mathrm{K}\right)$

$\mathrm{H} \quad$ jet throat height, $\mathrm{m}$

L average length of column of fluid ejected by

the jet in the blowing phase, $\mathrm{m}$

$\mathrm{Nu} \quad$ Nusselt number, dimensionless

$\mathrm{P}$ average pressure, $\mathrm{N} / \mathrm{m}^{2}$

Re Reynolds number, dimensionless

S deformation tensor, $1 / \mathrm{s}$

St Strouhal number, dimensionless

$\mathrm{t}$ time, $\mathrm{s}$

$\mathrm{T}$ average fluid temperature, $\mathrm{K}$

$\mathrm{u}$ time-varying jet velocity, $\mathrm{m} / \mathrm{s}$

$\mathrm{U}$ average flow velocity, $\mathrm{m} / \mathrm{s}$

$\mathrm{x} \quad$ Cartesian direction, $\mathrm{m}$

\section{Greek symbols}

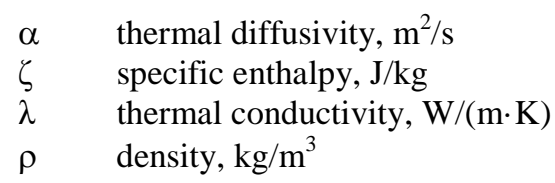

$$
\begin{array}{ll}
\theta & \text { dimensionless temperature } \\
\tau & \text { period of oscillation, } \mathrm{s} \\
\mu & \text { dynamic viscosity, } \mathrm{Pa} \cdot \mathrm{s} \\
\omega & \text { instantaneous voritcity, } 1 / \mathrm{s}
\end{array}
$$

\section{Subscripts

$\begin{array}{ll}\mathrm{i}, \mathrm{j} & \text { indices for Cartesian directions } \\ 0 & \text { average jet property } \\ \mathrm{t} & \text { turbulent properties } \\ \mathrm{T} & \text { total property }\end{array}$

\section{INTRODUCTION}

The constant advance in electronic design results in higher core densities, which increase the amount of power being consumed and heat generation. This combination of higher power and smaller scale results in high heat fluxes that must be removed from the electronic components in order to avoid failure by overheating. Despite higher thermal loads, temperature limits of electronic components have remained at around $85^{\circ} \mathrm{C}$ to ensure its operation and durability (Bhowmik et al., 2005, Mathews and Balaji, 2006). Consequently, new and more efficient forms of heat dissipation are needed to cope with the rising cooling demand (Chaudhari et al., 2010, Chandratilleke et al., 2010, Kalteh et al., 2011).

In order to cope with this higher heat flux, new 
heat dissipation techniques, such as micro channels, micro heat pipes, thermoelectric coolers, or liquid cold plates have been considered (Etemoglu, 2007). Overall, liquid cooling is considered more readily capable to handle the temperatures of high power systems. However, as noted by Xu et al. (1998), air is still widely used as the working fluid in electronic cooling due to its negligible cost and availability. Additionally, heat removed from the vicinity of the electronic component must still be discharged to the surroundings, and traditionally this task is still accomplished by finned heat sinks and conventional fans. Thus, despite its limitations, forced air convection still remains the least expensive, widely used and more reliable form of cooling. The downside of forced air convection, as observed by Chaudhari et al. (2010b), is the need to provide a large volume of air to create a pressure difference sufficient to flow through the narrow network of channels and fins that make up a heat sink and remove heat at a reasonable rate.

Therefore, any technique that can enhance heat transfer for the current amount of airflow available is worthy of study (Ohadi, 2003). For this reason, synthetic jets are viewed with great interest due to their known capability to enhance mixing or manipulate turbulence. Additionally, synthetic jets lack the need for an external source of fluid and have the potential for miniaturization, which allow the fabrication of such devices embedded directly alongside the electronic components (Chaudhari et al., 2010a).

Most cooling studies with synthetic jets make use of a direct impact configuration, in which the synthetic jet actuator is placed facing a heated surface and the jet collides head-on against the heating element. However, Mahalingan and Glezer (2005) opted to use a different configuration in which the synthetic jet is directed to blow tangentially to one of the walls of a channel representing the surface of a fin. Under continuous operation, the effect of the synthetic jet was to induce a mean flow through the channel. Experimental results show that the convective heat transfer coefficient of the synthetic jet was $150 \%$ higher than for a conventional steady channel flow of equivalent mean mass flowrate.

Thus, the objectives of this study are to quantify the heat transfer capabilities of this chosen configuration with regards to the jet velocity, pulsing frequency and position of the heated element. The chosen methodology is of a numerical simulation, which allows the execution of studies over several parametric variables of interest.

\section{Synthetic Jets}

A synthetic jet is formed when fluid is alternately ejected and suctioned into a cavity through an orifice. The usual configuration of a synthetic jet actuator consists of a cavity sealed on end with a flexible membrane and on the other end with a plate in which an orifice is cut out (Glezer et al., 2003). Figure 1 shows a schematic of a synthetic jet generator and the ensuing jet. As it can be seen on Fig. 1, a synthetic jet actuator does not require an external source of fluid, such as a feed tank of pressurized air, since the same fluid in external region also fills the cavity (Glezer et al., 2003). However, despite the zero mass flow balance of fluid in and out of the cavity, a net positive momentum develops in the external region away from the jet orifice with average characteristics similar to a steady jet (Chaudhari et al., 2010a; Jain et al., 2011).

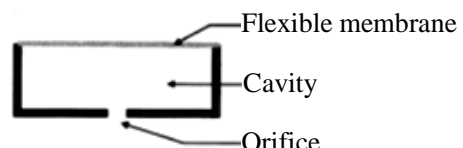

(a)

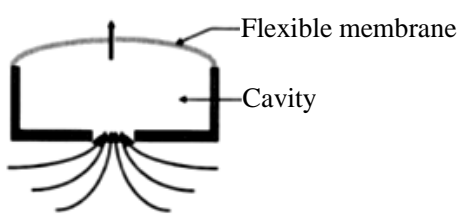

(b)

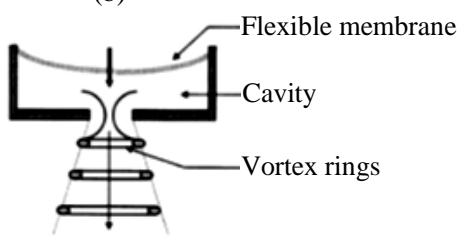

(c)

Figure 1. Schematic of a synthetic jet actuator and its operation: (a) basic components; (b) suction phase;

(c) ejection phase and formation of synthetic jet.

Source: Adapted from Glezer et. al, (2003).

The synthetic jet is formed during the ejection phase of the actuator, as fluid rolls up on the shear layer at the edge of the orifice to form a vortex pair or vortex ring. This vorticity is the driving mechanism behind the enhanced convection and turbulence caused by the jet. As noted by Smith and Glezer (1998), individual, well-formed vortices are observed in region near the jet orifice. However, these vortices quickly break down into a turbulent jet within a few orifice diameters of distance. While the production of vorticity also occurs on steady jets, the oscillatory nature of the synthetic jet augments this phenomenon considerably. For the correct operation of a synthetic jet, the ejected vorticity must contain sufficient celerity so that it is not re-ingested into the cavity when the actuator reverses direction. If these conditions are met, the suction phase draws fluid into the cavity not from the main jet but from along the sides of the orifice in the external medium (Williams et al., 2007).

Chaudhari et al. (2009) analyzed the effect of pulsing frequency on the ensuing jet for different 
cavity depths and orifice diameters. Experiments show that the synthetic jet reaches peak velocity values within a narrow frequency range. Moreover, two critical frequencies were identified within this frequency range at which the velocity peaked. These values were identified as the resonant frequency of the diaphragm and the Helmholtz frequency of the cavity. Additionally, Chaudhari et al. (2009) noted that the operating frequency range became narrower with increasing orifice diameter and the cavity depth has a more pronounced effect only for smaller orifice diameters and at the Helmholtz frequency.

\section{Governing Equations}

The governing equations are the conservation of mass, conservation of momentum and energy equation applied to an incompressible fluid with constant thermal properties in a two-dimensional domain. In index notation, these equations are given respectively as:

$$
\frac{\partial \mathrm{U}_{\mathrm{i}}}{\partial \mathrm{x}_{\mathrm{i}}}=0
$$

$$
\begin{aligned}
\frac{\partial \rho U_{i}}{\partial t}+ & \frac{\partial}{\partial x_{j}}\left(\rho U_{i} U_{j}\right)= \\
& -\frac{\partial P}{\partial x_{i}}+\frac{\partial}{\partial x_{j}}\left[\left(\mu+\mu_{t}\right) S_{i j}\right] ;
\end{aligned}
$$

$$
\begin{aligned}
& \frac{\partial \rho \zeta_{\mathrm{T}}}{\partial \mathrm{t}}-\frac{\partial \mathrm{P}}{\partial \mathrm{t}}+\frac{\partial}{\partial \mathrm{x}_{\mathrm{j}}}\left(\rho \mathrm{U}_{\mathrm{j}} \zeta_{\mathrm{T}}\right)= \\
& \frac{\partial}{\partial \mathrm{x}_{\mathrm{j}}}\left[\left(\lambda+\rho \mathrm{C}_{\mathrm{P}} \alpha_{\mathrm{t}}\right) \frac{\partial \theta}{\partial \mathrm{x}_{\mathrm{j}}}\right] \\
&+\frac{\partial}{\partial \mathrm{x}_{\mathrm{j}}}\left[\mathrm{U}_{\mathrm{i}}\left(\mu+\mu_{\mathrm{t}}\right) \mathrm{S}_{\mathrm{ij}}\right] .
\end{aligned}
$$

In Eq. (1) through (3), $U$ is the mean flow velocity, $\mathrm{m} / \mathrm{s}$; $\mathrm{x}$ is a Cartesian direction, $\mathrm{m}$; $\mathrm{i}$ and $\mathrm{j}$ are indices for each directions in the domain; $\rho$ is the density, $\mathrm{kg} / \mathrm{m}^{3}$; $\mathrm{t}$ is the time, $\mathrm{s} ; \mathrm{P}$ is the pressure, $\mathrm{N} / \mathrm{m}^{2} ; \mu$ and $\mu_{\mathrm{t}}$ are the dynamic and turbulent viscosities, respectively, $\mathrm{Pa} \cdot \mathrm{s}$; $\mathrm{S}_{\mathrm{ij}}$ is the deformation tensor of the fluid element, $1 / \mathrm{s} ; \zeta_{\mathrm{T}}$ is the total specific enthalpy, $\mathrm{J} / \mathrm{kg} ; \lambda$ is the fluid thermal conductivity, $\mathrm{W} /(\mathrm{m} \cdot \mathrm{K}) ; \mathrm{C}_{\mathrm{p}}$ is the fluid specific heat at constant pressure, $\mathrm{J} /(\mathrm{kg} \cdot \mathrm{K}) ; \alpha_{\mathrm{t}}$ is the turbulent thermal diffusivity $\mathrm{m}^{2} / \mathrm{s}$; and $\theta$ is a dimensionless temperature.

The governing equations were part of the ANSYS CFX software package and turbulent closure was provided by the SST k- $\omega$ model. This turbulent model was selected since it is recommended for walldriven flows with adverse pressure gradient separation. As noted by Menter (2003), this model combines the versatility of the $\mathrm{k}-\varepsilon$ and precision of the $k-\omega$ models without the artificial accumulation of turbulence in stagnation regions.

\section{Computational Domain}

The geometry studied consists of a 2-D channel filled with incompressible fluid as seen on Figure 2. The lower surface is defined as a no-slip wall, while the left and right borders are defined as openings at the same static pressure and initially with no mass flow. The top surface is defined as a symmetry condition, so that the computational domain is essentially the bottom half of a symmetric design. A thin plate is placed at a height $\mathrm{H}$ above the lower surface to form the throat for the synthetic jet actuator. This geometry was constructed to represent the narrow space between fins in an electronic heat sink cooler.

The throat length was made to be excessively long, at least $25 \mathrm{H}$ so that jet formation would not be affected by the pressure condition at the left opening. The long throat length also precludes the need to simulate the cavity and membrane of the synthetic jet actuator. A sample geometry, which included the cavity membrane and a connecting duct to the jet throat was tested and showed no significant difference in the throat flow. As a matter of fact, the throat flow exhibited typical characteristics of an incompressible, oscillating duct flow. Experimental studies such as Chaudhari et al. (2009) also noted that jet velocities were fairly insensitive to the depth of the cavity. Thus, it was possible to replace the cavity and membrane with a shaped, time-varying velocity profile applied directly at the throat inlet, and the flow allowed to develop naturally along the throat length until it is discharged in the channel. Based on the orifice diameter $\mathrm{H}$, the channel has an overall length of $200 \mathrm{H}$ and height of $9.5 \mathrm{H}$.

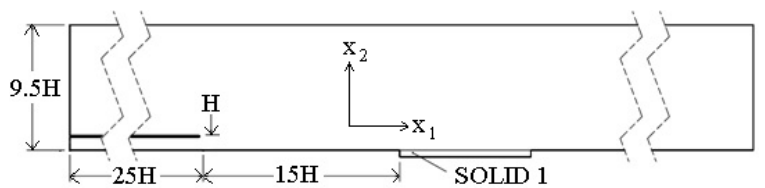

(a)

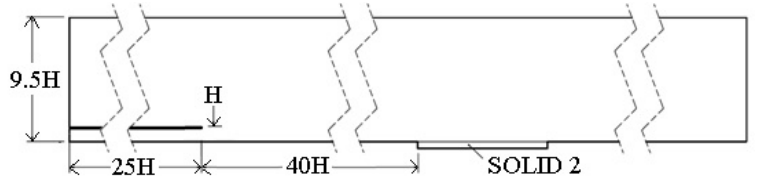

(b)

Figure 2. Schematic of channel geometries used in this study for configuration (a) Solid 1 and (b) Solid.

For the heat transfer analysis, a heated element representing an electronic chip measuring $10 \mathrm{H}$ in length and a depth of $0.5 \mathrm{H}$ is embedded on the lower surface. As seen on Fig. 2, two locations are 
considered based on the distance between the jet orifice exit plane and the start of the heated element: Solid 1(Fig. 2(a)) has the heated element placed at a distance of $15 \mathrm{H}$ from the jet orifice while Solid 2 (Fig. 2(b)) has the heated element placed at a distance of $40 \mathrm{H}$. Vertical grid refinement is applied near noslip walls to ensure sufficient resolution to capture boundary layer phenomena. As seen in Figure 3, in addition to vertical grid refinement, horizontal grid refinement is also applied at regions where larger gradients are expected to occur, or where additional resolution is deemed beneficial. These regions are identified to be around the jet orifice exit plane, upstream and downstream of the heated region. Overall, the computational meshes used in this study contain around 90,000 elements and 180,000 nodes.

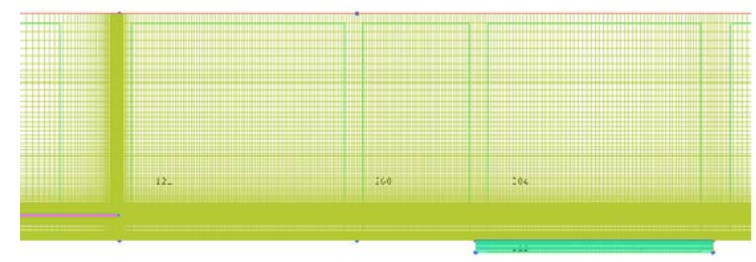

Figure 3. Section of computational mesh showing grid refinement near walls and regions of interest.

The current grid and numerical method was tested with the computational domain without the raised plate that forms the jet throat. Thus the geometry was essentially that of a channel flow. A pressure gradient was specified between the left and right openings and a turbulent channel flow allowed to develop until a steady state condition is reached. The jet profile near channel exit was extracted and the result compared to the universal turbulent boundary layer profile. The extracted velocity was found to be well matched to the theoretical profile of White (1991), containing all expected regions: laminar sublayer, overlap region and log-law region. The resulting mass flow rate is applied as a boundary condition and the resulting pressure gradient and developed velocity profile were found to be the same as the initial test. This mesh resolution was kept throughout the remainder of this study. Since all other simulations resulted in much inferior average mass flow rates through the channel than in the validation cases, the computational mesh was deemed to be sufficient converged to obtain accurate results of the resulting turbulent structures.

\section{Flow and Simulation Parameters}

The main parameters of interest for this study are the Strouhal number, Reynolds number and Nusselt number. The Strouhal number (St) is used to characterize oscillating flows, where it functions as a dimensionless measure of frequency. Its application in synthetic jets is to serve as a limiting actuation value, above which no synthetic jet is issued from the orifice. The mathematical expression of St used in this study is given by

$$
\mathrm{St}=\frac{2 \pi \mathrm{fH}}{\mathrm{U}_{0}},
$$

where $\mathrm{f}$ is the pulsing frequency of the jet, s; and $\mathrm{H}$ is the jet orifice throat height, $\mathrm{m}$. The average exit jet velocity, $\mathrm{U}_{0}$, is defined as

$$
\mathrm{U}_{0}=\frac{\mathrm{L}_{0}}{\tau},
$$

where $\tau$ is the period of oscillation, $\mathrm{s}$; and $\mathrm{L}_{0}$ is the average length of a column of fluid ejected during the blowing phase of the jet, defined by

$$
\mathrm{L}_{0}=\int_{0}^{\tau / 2} \mathrm{u}_{0}(\mathrm{t}) \mathrm{dt}
$$

where $u_{0}$ is the time-varying velocity applied as a boundary condition at the throat entrance. This velocity is calculated assuming a sinusoidal velocity profile at the throat entrance,

$$
\mathrm{u}_{0}(\mathrm{t})=\mathrm{A}_{0} \sin \left(\frac{2 \pi \mathrm{t}}{\tau}\right) \int_{0}^{\mathrm{H}} \sin \left(\frac{\pi \mathrm{y}}{\mathrm{H}}\right) \mathrm{dx}_{2},
$$

where $\mathrm{A}_{0}, \mathrm{~m} / \mathrm{s}$, denotes an amplitude of oscillation of the velocity at the throat entrance region; and $x_{2}$ is the transversal direction with respect to the channel length as shown on Fig. 1.

The Reynolds number ( $\mathrm{Re}$ ) is defined according to the average jet velocity and orifice height, being defined as

$$
\operatorname{Re}=\frac{\rho \mathrm{U}_{0} \mathrm{H}}{\mu},
$$

where $\mu$ is the dynamic viscosity of the fluid, $\mathrm{Pa} \cdot \mathrm{s}$.

For problems relating to heat transfer, the average Nussel number $(\mathrm{Nu})$ over the heated element becomes an important analysis parameter, being defined as

$$
\mathrm{Nu}=\frac{\mathrm{hd}}{\lambda}
$$

where $h$ is the average convective heat transfer coefficient, $\mathrm{W} /\left(\mathrm{m}^{2} \cdot \mathrm{K}\right)$; and $\mathrm{d}$ is a characteristic length of the surface, $\mathrm{m}$, in this case the length of the heated element.

A baseline case was defined with amplitude $\mathrm{A}_{0}$ $=0.755 \mathrm{~m} / \mathrm{s}$, which results in a value of $\mathrm{U}_{0}$ that is half 
of the value used in the turbulent jet of Smith and Glezer (1998). From this baseline case, the amplitudes of velocity corresponding to (1/2) $A_{0}$ and $(3 / 2) A_{0}$ are also examined. The range of Reynolds number tested in this study is shown on Table 1.

Table 1. Amplitudes of oscillation tested and resulting average exit jet velocity $\left(\mathrm{U}_{0}\right)$ and Reynolds numbers (Re).

\begin{tabular}{|l|c|c|}
\hline Amplitude & $\mathrm{U}_{0}(\mathrm{~m} / \mathrm{s})$ & $\mathrm{Re}$ \\
\hline $0.5 \mathrm{~A}_{0}$ & 0.077 & 85.8 \\
\hline $1.0 \mathrm{~A}_{0}$ & 0.153 & 172 \\
\hline $1.5 \mathrm{~A}_{0}$ & 0.230 & 257 \\
\hline
\end{tabular}

Two values of pulsing frequency (f) are also considered, case St1 with $\mathrm{f}=1 \mathrm{~Hz}$ and case St2 with $\mathrm{f}$ $=0.5 \mathrm{~Hz}$. These values are considerably lower than experimental studies but result in values of Strouhal number within the range needed to induce the formation of vorticity. Consequently, they are considered sufficient to obtain a measure of the effect of pulsing frequency on jet cooling. The range of St tested is shown on Table 2 .

Table 2. Strouhal numbers (St) for each pulsing frequency (f) and average jet velocity $\left(\mathrm{U}_{0}\right)$.

\begin{tabular}{|l|c|c|}
\hline $\mathrm{U}_{0}[\mathrm{~m} / \mathrm{s}]$ & $\mathrm{St} 1(1 \mathrm{~Hz})$ & $\mathrm{St} 2(0.5 \mathrm{~Hz})$ \\
\hline 0.077 & 0.082 & 0.164 \\
\hline 0.153 & 0.041 & 0.082 \\
\hline 0.230 & 0.027 & 0.055 \\
\hline
\end{tabular}

\section{Numerical Results}

The variations in Re and St shown on Tab. 1 and Tab. 2 are applied to both Solid 1 and Solid 2 configurations. Initial conditions consisted of fluid at rest in the channel at a uniform temperature of $290 \mathrm{~K}$. At the start of jet actuation, a constant temperature of $300 \mathrm{~K}$ is applied as a boundary condition along the embedded bottom of the heating element and heat is allowed to flow through the solid region and into the fluid. The fluid and solid elements were defined so that the thermal conductivity of the solid is 690 times greater than the fluid. Jet actuation induces the formation of a mean flow in the channel along the direction $\mathrm{x}_{1}$ of Fig. 1. As fluid oscillates downstream along the channel, fluid that is drawn in and out through the left and right openings of the channel is also assumed to be at $290 \mathrm{~K}$. For each run, the specific value of $\mathrm{U}_{0}$ and the outside temperature of $290 \mathrm{~K}$ were used as normalization parameters for momentum and thermal results, respectively.

Each synthetic jet case was allowed to run through 10 pulsing cycles, at which point the mean flow of the channel stabilized. Time-averaged velocity and temperature fields were calculated over the $10^{\text {th }}$ pulsing cycle. The average heat flux and the average surface temperature were calculated along the length of the heated element in order to obtain the average convective heat transfer coefficient.
For comparison purposes, the average mass flow rate in the channel induced by the synthetic jet actuation was calculated. To simulate the effect of a conventional cooler, a new simulation with the same initial conditions was tested but with the synthetic jet inoperative and a uniform velocity profile applied at the left entrance of the channel which resulted in an average mass flow identical to the synthetic jet. The simulation was allowed to reach a steady state solution and the average flow and thermal properties were calculated. This procedure was repeated for each case tested so that in total, 24 simulations were conducted.

Since each synthetic jet case is compared to its respective steady channel case, a direct comparison can be made of the heat dissipation produced by the synthetic jet and a conventional flow in which a large single cooler fan drives a steady volume of air through a heat sink. Table 3 shows heat transfer results for the heated element placed in the Solid 1 configuration with both $1 \mathrm{~Hz}$ (St1) and $0.5 \mathrm{~Hz}$ (St2) pulsing frequencies. Table 4 shows heat transfer results for the heated element placed in the Solid 2 configuration with both $1 \mathrm{~Hz}$ (St1) and $0.5 \mathrm{HZ}$ (St2) pulsing frequencies. The synthetic jet results are listed as "Jet" and the equivalent conventional cooler fan result is listed as "Cooler."

Table 3(a). Heat transfer results for Solid 1 and St1.

\begin{tabular}{|l|c|c|c|c|c|}
\hline \multirow{2}{*}{$\operatorname{Re}$} & \multicolumn{2}{|c|}{$\mathrm{h}\left[\mathrm{W} /\left(\mathrm{m}^{2} \cdot \mathrm{K}\right)\right]$} & & \multicolumn{2}{|c|}{$\mathrm{Nu}$} \\
\cline { 2 - 3 } \cline { 5 - 5 } & Jet & Cooler & Jet & Cooler \\
\hline 85.8 & 1830 & 960 & & 30.2 & 15.8 \\
\hline 172 & 3730 & 2070 & 61.5 & 34.0 \\
\hline 257 & 9610 & 4350 & & 158 & 71.7 \\
\hline
\end{tabular}

Table 3(b). Heat transfer results for Solid 1 and St2.

\begin{tabular}{|c|c|c|c|c|}
\hline \multirow{2}{*}{ Re } & \multicolumn{2}{|c|}{$\mathrm{h}\left[\mathrm{W} /\left(\mathrm{m}^{2} \cdot \mathrm{K}\right)\right]$} & \multicolumn{2}{|c|}{$\mathrm{Nu}$} \\
\hline & Jet & Cooler & Jet & Cooler \\
\hline 85.8 & 1690 & 903.5 & 27.9 & 15.0 \\
\hline 172 & 3580 & 2020 & 59.0 & 33.3 \\
\hline 257 & 8940 & 4280 & 147 & 70.5 \\
\hline
\end{tabular}

Table 4(a). Heat transfer results for Solid 2 and St1.

\begin{tabular}{|c|c|c|c|c|}
\hline \multirow{2}{*}{$\operatorname{Re}$} & \multicolumn{2}{|c|}{$\mathrm{h}\left[\mathrm{W} /\left(\mathrm{m}^{2} \cdot \mathrm{K}\right)\right]$} & \multicolumn{2}{|c|}{$\mathrm{Nu}$} \\
\hline & Jet & Cooler & Jet & Cooler \\
\hline 85.8 & 1450 & 1020 & 23.9 & 16.8 \\
\hline 172 & 3460 & 2217 & 57,0 & 36.8 \\
\hline 257 & 9520 & 4280 & 157 & 70.5 \\
\hline
\end{tabular}

Table 4(b). Heat transfer results for Solid 2 and St2.

\begin{tabular}{|c|c|c|c|c|}
\hline \multirow{2}{*}{$\operatorname{Re}$} & \multicolumn{2}{|c|}{$\mathrm{h}\left[\mathrm{W} /\left(\mathrm{m}^{2} \cdot \mathrm{K}\right)\right]$} & \multicolumn{2}{|c|}{$\mathrm{Nu}$} \\
\hline & Jet & Cooler & Jet & Cooler \\
\hline 85.8 & 1440 & 921 & 23.8 & 15.2 \\
\hline 172 & 3490 & 2150 & 57.6 & 35.4 \\
\hline 257 & 8950 & 4190 & 148 & 69.0 \\
\hline
\end{tabular}

Results from Tab. 3 and Tab. 4 are shown in graphic format as Figures 4 and 5. Figures 4 and 5 show that synthetic jet-driven cooling outperforms 
conventional steady coolers for every single combination of Re, St and placement of heated region. The improvement in $\mathrm{Nu}$ varied from a minimum of $40 \%$ to as high as $220 \%$. Overall, heat transfer tends to increase with increasing Re. The gains themselves are not exactly proportional. Starting with a Re of 85.8 and doubling its value to 172 results in increases in $\mathrm{h}$ and $\mathrm{Nu}$ in factors ranging from 2 to 2.8. If this initial Re is increased by a factor of 3 to 257, h and Nu increase in factors ranging from 5.2 to 6.2 . These gains occur to both the synthetic, jet driven cooling and the conventional steady channel flow. Placing the heated region closer to the jet orifice resulted in the Solid 1 configuration having higher $\mathrm{Nu}$ (23\% for St1 and 18\% for St2) than for the Solid 2 configuration for the lowest Re. As Re increases, the values of $\mathrm{Nu}$ converged towards very similar values for both configuration, indicating that heated element placement becomes less influential in cooling. For each configuration, pulsing frequency did not affect substantially $\mathrm{Nu}$ at lower Re. Only at the highest Re tested, the St1 case resulted in a slightly higher Re (7.5\%) than for the St2 case.

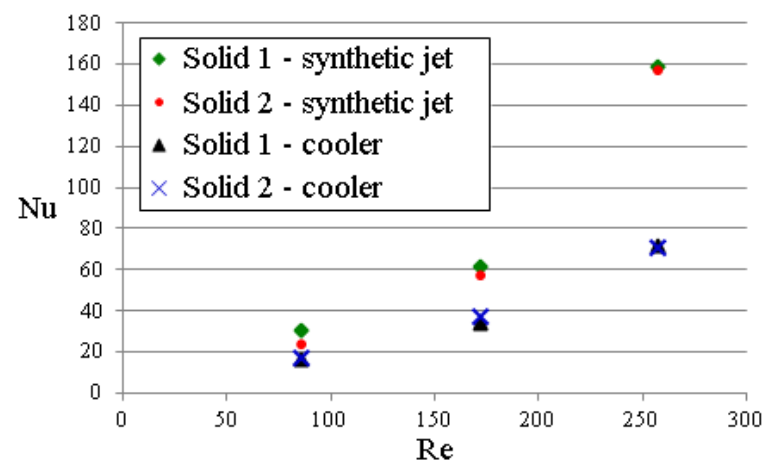

Figure 4. Nu vs. Re for Solid 1 and Solid 2 configurations and $\mathrm{f}=1 \mathrm{~Hz}$ (St1).

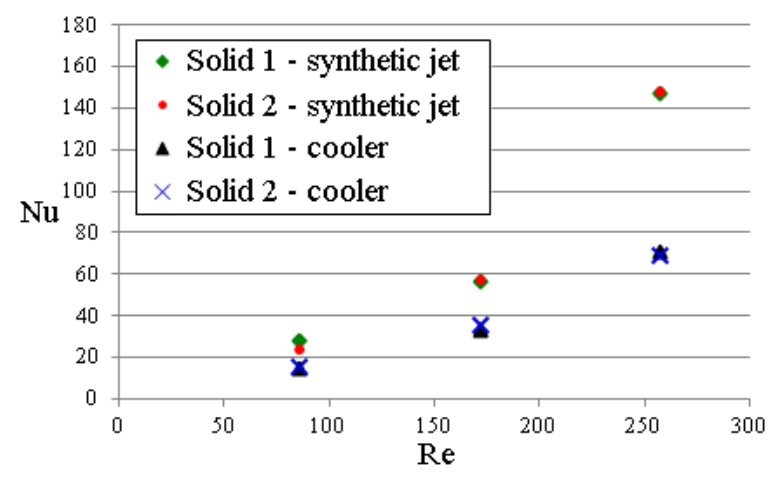

Figure 5. Nu vs. Re for Solid 1 and Solid 2 configurations and $\mathrm{f}=0.5 \mathrm{~Hz}$ (St2).

Further physical insight on the cooling effects of the synthetic jet can be obtained from the normalized instantaneous contours of vorticity of Figure 6. Fig. 6(a) and (b) compares the vorticity layer produced by the synthetic jet with the equivalent conventional cooler flow for the Solid 1 configuration, while Fig 6(c) and (d) perform the same comparison for the Solid 2 configuration.

Figure 6 show that for both configurations the jet actuator produced a thin layer of vorticity on the bottom wall of the channel. In comparison, the conventional cooler steady channel flow develops a thicker, but less intense vorticity layer along the same wall. This noticeable increase in vorticity and its associated turbulent mixing is responsible for the greatly superior cooling performance of the synthetic jet. The increase in cooling performance of the Solid 1 configuration at lower Re can be explained by the presence of larger, well-defined, rotating structures in the near jet region of the jet before its breakdown into smaller turbulent structures at further distances. As the $\mathrm{Re}$ increases, jet vorticity breakdown occurs earlier and placement of the heated element becomes less of a factor in cooling.
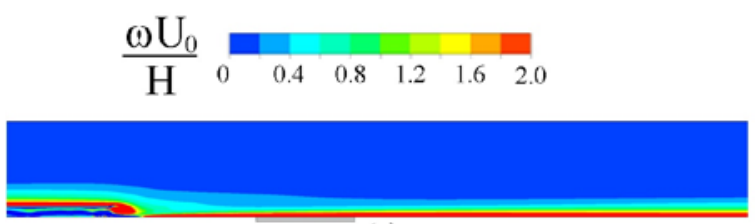

(a)

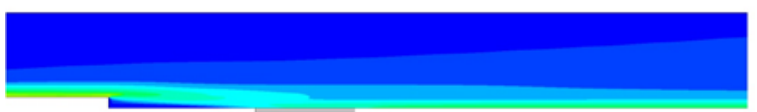

(b)

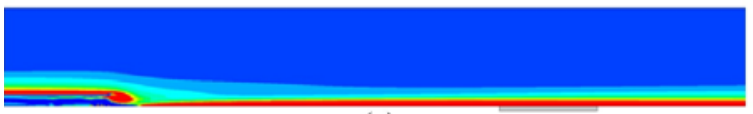

(c)

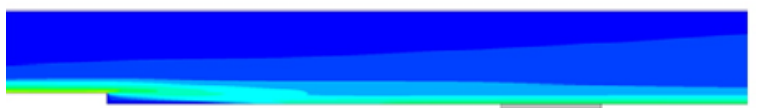

(d)

Figure 6. Normalized instantaneous contours of vorticity for $\mathrm{Re}=172$ and $\mathrm{f}=1 \mathrm{~Hz}$ (St1) for (a) Solid 1 at the at the $10^{\text {th }}$ pulsing cycle of the synthetic jet; (b) Solid 1 steady state solution with cooler; (c) Solid 2 at the at the $10^{\text {th }}$ pulsing cycle of the synthetic jet;

(b) Solid 2 steady state solution with cooler.

\section{CONCLUSIONS}

In this work, a numerical approach was conducted to demonstrate the cooling effectiveness of a synthetic jet directed to blow tangentially to a surface containing a heating element. The geometry chosen was representative of the narrow space between the fins of a computer heat sink cooler.

Initial studies show that, for an incompressible fluid and long connectors and throats between the synthetic jet generator and the jet orifice, the resulting flow resembles an oscillating duct flow. Therefore, the jet generator cavity and membrane can be replaced by an oscillating velocity boundary condition.

When compared to a conventional cooler 
producing a steady flow with identical mass flow rate as induced by the synthetic jet, tangential synthetic jet cooling yielded superior results across all values of Re, St and geometric configurations tested. The improvement in Nu varied from $40 \%$ to $220 \%$.

Higher pulsing amplitude and consequently Re results in higher $\mathrm{Nu}$ for all synthetic jets. However pulsing frequency has little effect at lower Re but results indicate improved performance with lower frequencies at higher Re.

At the lowest Re, heated elements placed closer to the jet orifice resulted in moderately higher cooling effects due to the presence of larger coherent vorticity before its breakdown into smaller turbulent structures. However, for other values of Re, jet actuation produces a layer of vorticity that covers the entire surface. Since cooling applications would most likely involve higher Re, this result indicates that placement of the heated element is not a substantial factor and, furthermore, suggests that a tangential jet configuration would be suitable to cooling the entire surface of a fin.

\section{ACKNOWLEDGEMENTS}

The authors would like to acknowledge the support of $\mathrm{CNPq}$ through project $\mathrm{MCT} / \mathrm{CNPq} \mathrm{n}^{\circ}$ $70 / 2009$

\section{REFERENCES}

Bhowmik, H., Tso, C. P., Tou, K. W., and Tan, F. L., 2005, Convection Heat Transfer from Discrete Heat Sources in a Liquid Cooled, Applied Thermal Engineering, Vol. 25, No. 16, pp. 2532-2542.

Chandratilleke, T. T., Jagannatha, D., and Narayanaswamy, R., 2010, Heat Transfer Enhancement in Microchannels with Cross-Flow Synthetic Jets, International Journal of Thermal Sciences, Vol. 49, No. 3, pp. 504-513.

Chaudhari, M., Verma G., Puranik, B., and Agrawal, A., 2009, Frequency Response of a Synthetic Jet Orifice, Experimental Thermal and Fluid Science, Vol. 33, pp. 439-448.

Chaudhari, M., Puranik, B., and Agrawal, A., 2010a, Heat Transfer Characteristcs of Synthetic Jet Impingement Cooling, International Journal of Heat and Mass Transfer, Vol. 53, pp. 1057-1069.

Chaudhari, M., Puranik, B., and Agrawal, A., 2010b, Effect of Orifice Shape in Synthetic Jet Based Impingement Cooling, Experimental Thermal and Fluid Science, Vol. 34, pp. 246-256.

Etemoglu, A. B., 2007, A Brief Survey Economical Analysis of Air Cooling for Electronic Equipments, International Communications in Heat and Mass Transfer, Vol. 34, No.1, pp. 103-113.

Glezer, A., Allen, M. G., Brand, O., Lee, J. B., and Kercher, D. S., 2003, Microjet Cooling Devices for Thermal Management of Electronics, IEEE
Transactions on Components and Packaging Technologies, Vol. 26, No. 2, pp. 359-366.

Jain, M., Puranik, B., and Agrawal, A., 2011, A Numeral Investigation of Effects os Cavity and Orifice Parameters on the Characteristics of a Synthetic Jet Flow, Sensors and Actuators A: Physical, Vol. 165, No. 2, pp. 351-366.

Kalteh, M., Abbassi, A., Saffar-Avval, M., Frijns, A., Darhuber, A., and Harting, J., 2011, Experimental and Numeral Investigation of Nanofluid Forced Convection Inside a Wide Microchannel Heat Sink, Applied Thermal Engineering, Vol. 36, pp. 260-268.

Mahalingam, R., and Glezer, A., 2005, Design and Thermal Characteristics of a Synthetic Jet Ejector Heat Sink Journal of Electronic Packaging, Vol. 127, No. 1, pp. 172-177.

Mathews, R. N., and Balaji, C., 2006, Numeral Simulation of Conjugate, Turbulent Mixed Convection Heat Transfer in a Vertical Channel with Discrete Heat Sources, International Communications in Heat and Mass Transfer, Vol. 33, No. 7, pp. 908916.

Menter, F. R., Kuntz, M., and Langtry, R., 2003, Ten Years of Industrial Experience with the SST Model, in: Turbulence, Heat and Mass Transfer IV, Antalya, Turkey.

Ohadi, M., 2003, Thermal Management of next Generation Low Volume Complex Electronics, in: Advanced Liquid Cooling, Scottsdale, AZ.

Smith, B. L., and Glezer, A., 1998, The Formation and Evolution of Synthetic Jets, Physics of Fluids, Vol. 10, No. 9, pp. 2281-2297.

White, F., 1991, Viscous Fluid Flow, 2nd Edition, McGraw-Hill.

Xu, G. P., Tou, K. W., and Tso, C. P., 1998, Numeral Modeling of Turbulent Heat Transfer from Discrete Heat Sources in Liquid-Cooled Channel, International Journal of Heat and Mass Transfer, Vol. 41, No. 10, pp. 1157-1166. 